\title{
PRÁTICAS DE ATIVIDADE FÍSICA E ESPORTE NO BRASIL
}

\section{Practice of physical activity and sports in Brazil \\ Prácticas de actividad física y deporte en Brasil}

\author{
Sarah Souza Pontes (iD \\ Universidade Federal da Bahia - UFBA - Salvador (BA) - Brasil
}

Alana das Mercês Silva (iD

Universidade Federal da Bahia - UFBA - Salvador (BA) - Brasil

Laíza Muniz de Santana Santos iD

Universidade Federal da Bahia - UFBA - Salvador (BA) - Brasil

Brendo Vitor Nogueira Sousa (iD

Universidade Federal do Recôncavo da Bahia - UFRB - Santo Antônio de Jesus (BA) - Brasil

Elenilda Farias de Oliveira (iD

Faculdade Adventista da Bahia - FADBA - Cachoeira (BA) - Brasil

\section{RESUMO}

Objetivo: Caracterizar os praticantes de esporte e atividade físicas no Brasil por meio da estratificação dos resultados da Pesquisa Nacional de Amostra por Domicílio. Métodos: Trata-se de estudo ecológico de análise estatística descritiva, realizada com dados secundários em nível nacional por meio da Pesquisa Nacional de Amostra por Domicílio do ano de 2015, com 71.142 mil pessoas de 151.189 mil unidades domiciliares distribuídas por todas as Unidades da Federação. Realizou-se análise estatística descritiva, sendo organizadas variáveis qualitativas em tabelas e gráficos, através do teste qui-quadrado, considerando $p \leq 0,05$. Resultados: Constatou-se o Distrito Federal como o estado que mais realiza atividade física no país (30,9\%) e o Mato Grosso o que menos realizou no período estudado $(8,4 \%)$. Em relação ao esporte, Alagoas encontra-se como o estado que menos praticou esporte (17\%) e o Amazonas o que mais o fez $(30,7 \%)$. Percebeu-se ainda uma relação positiva entre a prática de atividade física e do esporte e a renda per capita por domicílio evidenciando que, quanto maior a renda, maior a prática dessas atividades. Quanto ao estado civil, nota-se que os autodenominados solteiros são os que mais praticam ambas as modalidades (esporte $27 \%$ e atividade física $18 \%$ ), com diferenças significativas. Conclusão: Os praticantes de esporte e atividade física no Brasil no ano de 2015 caracterizam-se por serem do sexo masculino, com maior nível de escolaridade e renda, solteiros e que buscavam essas atividades a fim de melhorar sua qualidade de vida e o bem-estar.

Descritores: Atividade Motora; Exercício; Esforço Físico.

\section{ABSTRACT}

Objective: To characterize the individuals who practice sports and physical activity in Brazil by stratifying the results of the 2015 National Household Sample Survey. Methods: This is an ecological descriptive study off national secondary data from the 2015 National Household Sample Survey conducted with 71,142 people of 151,189 households from all the Units of the Federation. Descriptive statistical analysis was performed, and the data were organized in tables and graphs using the chi-squared test with $p \leq 0.05$. Results: The Federal District is the state with the highest rate of physical activity in the country (30.9\%) and Mato Grosso is the state with the lowest rate (8.4\%). As for sports, Alagoas is the state with the lowest rate of sports practice (17\%) and Amazonas the state with the highest rate (30.7\%). There was also a positive relationship between the practice of physical activity and sports and per capita income per household, thus showing that the higher the income, the higher the rate of practice of these activities. With regard to marital status, self-reported single individuals were the ones who practiced both modalities the most (sports $27 \%$ and physical activity $18 \%$ ), with significant differences. Conclusion: The individuals who practiced sports and physical activity in Brazil in the year 2015 were mostly men, single, presented higher levels of education and income, and sought these activities in order to improve their quality of life and well-being.

Descriptors: Motor Activity; Exercise; Physical Exertion.

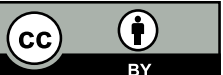




\section{RESUMEN}

Objetivo: Caracterizar las personas que hacen deporte y actividades físicas en Brasil a través de la estratificación de los resultados de la Investigación Nacional de Muestra por Domicilio. Métodos: Se trata de un estudio ecológico de análisis estadístico descriptivo realizado con datos secundarios de nivel nacional a través de la Investigación Nacional de Muestra por Domicilio del año de 2015 con 71.142 mil personas de 151.189 mil unidades domiciliarias distribuidas por todas las Unidades de la Federación. Se realizó un análisis estadístico descriptivo y las variables cualitativas han sido organizadas en tablas y gráficos a través de la prueba chi-cuadrado considerando $p \leq 0,05$. Resultados: Se constató que el Distrito Federal es el estado que más hace actividad física en el país (30,9\%) y el Mato Grosso el que menos ha realizado en el periodo investigado (8,4\%). Respecto al deporte, Alagoas es el estado que menos ha practicado deporte (17\%) y el Amazonas es el que más lo ha hecho (30,7\%). Se ha visto aún una relación positiva entre la práctica de actividad física y deporte y la renta per cápita por domicilio ha evidenciado que a mayor renta, mayor la práctica de esas actividades. Sobre el estado civil se nota que los que se denominan solteros son los que más hacen ambas modalidades (el deporte $27 \%$ y la actividad física 18\%) con diferencias significativas. Conclusión: Los que hicieron deporte y actividad física en Brasil en el año 2015 son del sexo masculino, con más escolaridad y renta, solteros y que buscaron esas actividades para mejorar su calidad de vida y el bienestar.

Descriptores: Actividad Motora; Ejercicio; Esfuerzo Físico.

\section{INTRODUÇÃO}

A globalização e o processo de urbanização ocasionaram diversas mudanças sociais, econômicas e epidemiológicas, dentre elas o aumento na expectativa de vida e consequentemente no desenvolvimento de doenças crônicas e degenerativas. $O$ crescente aumento dessas patologias tem apontado para o sedentarismo como um fator de risco para essas doenças, levando os sistemas de saúde pública a buscarem novas estratégias de promoção e prevenção da saúde, com foco na melhora da qualidade e hábitos de vida, o que inclui boa alimentação e prática de exercícios ${ }^{(1)}$.

Com essa mudança no perfil de adoecimento da população e uma maior valorização pelo corpo e saúde na sociedade contemporânea, existe uma grande tendência à prática de atividades físicas e esportes ${ }^{(1)}$. Isso porque a inatividade física, ou o baixo nível de atividade física, tem forte associação com as principais doenças crônicas que atingem a população mundial, tais como doenças cardiorrespiratórias (doença coronária, doenças cardiovasculares, acidente vascular cerebral, hipertensão), metabólicas (diabetes e obesidade), câncer (câncer de mama, câncer de cólon), musculoesquelética (osteoporose) e depressão(2). Ademais, o estilo de vida sedentário é o principal causador de mais de $50 \%$ dos casos de óbito no Brasil(3).

Com a mudança do conceito de saúde iniciada entre as décadas 70 e 80 e a criação do Sistema Único de Saúde (SUS), o incentivo à prática de atividades físicas tem aumentado tanto pelos programas de saúde pública como pela ampliação das práticas corporais e atividades físicas, promovido, principalmente, pela Política Nacional de Promoção da Saúde (PNPS), pela iniciativa própria e pela privada ${ }^{(4)}$.

Com o maior destaque para as estratégias de promoção e prevenção à saúde, a epidemiologia da atividade física tem tido destaque nas avaliações da saúde e nos processos relacionados à saúde. Para isso, são necessárias vigilância e monitorização dos fatores de risco fazendo o diagnóstico de cada território, para assim adotar medidas de promoção à saúde, prevenção e controle dessas doenças, desde que suas ações sejam voltadas para seus principais fatores de risco modificáveis ${ }^{(5)}$. Para a investigação da situação social e de saúde da população brasileira, é necessária a utilização de instrumentos e estratégias de busca dessas informações ${ }^{(6)}$.

Dentre esses instrumentos, no ano de 1967, foi criada a Pesquisa Nacional por Amostra de Domicílios (PNAD) com o objetivo de avaliar o desenvolvimento socioeconômico, obtendo informações estimadas em todo o país. A PNAD possui diversos questionários e alguns componentes específicos de saúde, executados nos anos de 1998, 2003 e 2008. No ano de 2015, a prática de atividade física foi avaliada pela primeira vez pelo Instituto Brasileiro de Geografia e Estatística (IBGE) ${ }^{(7)}$.

O PNAD 2015 além de permitir identificar o cenário demográfico e socioeconômico da população brasileira, por meio da caracterização de variáveis como sexo, idade, trabalho, renda, educação, situação do domicílio, fecundidade, entre ouras características, também investigou as práticas de esporte e atividade física em parceria com o Ministério do Esporte. Essas informações são de suma importância para que os profissionais de saúde e os serviços de saúde pública possam conhecer o nível de atividade física da população por região e, assim, criar estratégias de promoção e prevenção da saúde voltadas para esses grupos de acordo com suas necessidades ${ }^{(8)}$. 
Levando-se em consideração as informações supracitadas e a importância delas, este estudo tem como objetivo caracterizar os praticantes de esporte e atividade físicas no Brasil por meio da estratificação dos resultados da Pesquisa Nacional de Amostra por Domicílio.

\section{MÉTODOS}

Trata-se de um estudo ecológico que permite avaliar como o contexto social e ambiental podem afetar a saúde de grupos populacionais. Utilizou-se neste estudo dados secundários da PNAD 2015, instalado de forma gradativa no Brasil.

Cabe salientar que se incluíram na PNAD 2015 mais quatro pesquisas complementares, relacionadas às práticas de esporte e atividade física, acesso aos meios de comunicação (Internet, televisão, posse de telefone móvel celular para uso pessoal), questões dos cuidados das crianças menores de 4 anos de idade e aspectos relacionados ao trabalho e sindicalização( ${ }^{(8)}$.

Obteve-se a população do estudo por meio de amostra probabilística, selecionada em três estágios. As unidades primárias, representadas pelos municípios, categorizadas em autorrepresentativas e não autorrepresentativas. Sendo segmentados em grupos e cada um selecionado com probabilidade proporcional à população residente obtida no Censo Demográfico $2010^{(8)}$.

As unidades secundárias retratam os setores censitários, selecionadas em cada município da amostra, pela probabilidade proporcional obtida por meio dos dados de unidade domiciliar existentes do Censo Demográfico 2010 como medida de tamanho de amostra. As unidades terciárias representadas por domicílios, obtidas por meio da equiprobabilidade por unidade territorial da amostra, dos domicílios particulares e habitações em domicílios coletivos para análise das características dos moradores e da habitação.

Essa amostra é composta por 1.100 municípios, 151.189 unidades domiciliares, totalizando 356.904 pessoas que participaram da pesquisa. Para o tema suplementar Práticas de Esporte e Atividade Física, investigaram-se 71.142 mil pessoas acima de 14 anos, conforme os critérios estabelecidos pelo próprio PNAD ${ }^{(8)}$.

Tratando-se da investigação do tema suplementar Práticas de Esporte e Atividade Física, cabe salientar que para a seleção dos domicílios por amostragem aleatória e simples, considerou-se apenas os amostrados para o PNAD 2015. Importante ressaltar que houve diferença nos percentuais de domicílios selecionados, de acordo com a Unidade da Federação, a saber: Roraima e Amapá, 100\% dos domicílios da amostra da PNAD 2015 selecionados para o suplemento; Acre, Piauí Alagoas, Mato Grosso do Sul, Rio Grande do Norte e Tocantins, 80\%; nas outras Unidades da Federação, $60 \%$. Dessa forma, no último estágio, em cada domicílio da amostra, selecionou-se com equiprobabilidade, uma pessoa moradora de 15 anos ou mais de idade, para responder ao questionário ${ }^{(8)}$.

O questionário relacionado à prática de esporte e atividade física era composto por 34 perguntas de múltipla escolha, subdividido em 5 dimensões: para a pessoa que pratica esporte; característica do esporte único ou principal; para a pessoa que não praticou esporte no último ano; para a pessoa que praticou atividade física no último ano e característica da atividade física única ou principal(8).

Por meio do formulário objetivou-se identificar quem praticou esporte ou atividade física no período da pesquisa, bem como o tipo de modalidade esportiva e de atividade física; motivo que levou a realizá-lo; frequência; duração; local da prática entre outros fatores relacionados à sua atividade; bem como, estado civil, aspectos socioeconômicos e motivo desta prática. Salientando-se que não houve critérios para diferenciar esporte e atividade física, a pessoa investigada que classificou o tipo de atividade ${ }^{(8)}$.

Realizou-se análise estatística descritiva, sendo organizadas variáveis qualitativas em tabelas e gráficos, inferência estatística através do teste qui-quadrado, considerando-se $p \leq 0,05$, por meio do programa Statistical Package for the Social Sciences (SPSS) versão 22.0.

\section{RESULTADOS}

Percebeu-se que no ano de 2015, das 161,8 milhões de pessoas na faixa etária de 15 anos ou mais, 61,3 milhões (37,9\%) praticaram atividade física ou alguma modalidade esportiva.

Nas regiões Sul e Centro-Oeste, houve uma proporção $(41,1 \%)$ maior que a média nacional, enquanto as regiões Nordeste $(36,3 \%)$, Norte $(36,6 \%)$ e Sudeste $(37,5 \%)$ apontaram percentuais inferiores ${ }^{(8)}$.

Convêm informar que os dados do PNAD 2015 estão apresentados apenas em forma de porcentagem no documento original e em todas as suas referências, não estando disponíveis em valores absolutos. 
Na Tabela I estão expostos os dados referentes à prática de atividade física e esporte por estado e federação do Brasil. Tratando-se da prática de atividade física percebe-se que os estados que mais praticam são: no Norte, Roraima (22\%); no Nordeste, o Piauí $(26,1 \%)$ e a Paraíba (26,9\%); no Sudeste, São Paulo (19,9\%); no Sul, o Rio Grande do Sul $(27,5 \%)$ e no Centro Oeste, o Distrito Federal (30,9\%).

Sobre a realização de esportes, os estados com maiores índices, a saber, no Norte o Amazonas (30,7\%), no Nordeste o Ceará $(22,9 \%)$ e a Bahia $(22 \%)$, no Sudeste, Minas Gerais $(25,5 \%)$, no Sul, Santa Catarina $(28,2 \%)$ e no Centro Oeste o Distrito Federal (27,9\%), o Mato Grosso do Sul $(27,8 \%)$ e o Mato Grosso $(27,8 \%)$, Tabela I.

A Tabela II apresenta informações referentes aos motivos que levaram estas pessoas a realizarem atividades físicas e ou esportes de acordo com a renda per capita por domicílio e por sexo. Assim, evidencia-se que os principais motivos relatados em ambas as variáveis se basearam em manter a qualidade de vida e ou bem-estar, melhorar e ou manter o desempenho físico e para relaxar e ou se divertir. Também é possível observar uma relação positiva entre a prática de atividade física e de esporte e a renda per capita por domicílio, evidenciando que quanto maior a renda, maior a adoção desses hábitos.

Tabela I - Prática de atividade física e esporte no Brasil por estado e federação. Dados proporcionais de 71.142 brasileiros. Salvador, Bahia, 2015.

\begin{tabular}{|c|c|c|c|c|}
\hline \multirow[t]{2}{*}{ Estado e federação } & \multicolumn{2}{|c|}{ Atividade Física (\%) } & \multicolumn{2}{|c|}{ Esporte (\%) } \\
\hline & Sim & Não & Sim & Não \\
\hline \multicolumn{5}{|l|}{ Norte } \\
\hline Rondônia & 15,3 & 84,7 & 18,4 & 81,6 \\
\hline Acre & 18,6 & 81,4 & 20,3 & 79,7 \\
\hline Amazonas & 13,7 & 86,3 & 30,7 & 69,3 \\
\hline Roraima & 22,0 & 78,0 & 22,8 & 77,2 \\
\hline Pará & 12,4 & 87,6 & 24,7 & 75,3 \\
\hline Amapá & 15,6 & 84,4 & 22,7 & 77,3 \\
\hline Tocantins & 14,7 & 85,3 & 21,7 & 78,3 \\
\hline \multicolumn{5}{|l|}{ Nordeste } \\
\hline Maranhão & 20,7 & 79,3 & 19,9 & 80,1 \\
\hline Piauí & 26,1 & 73,9 & 19,3 & 80,7 \\
\hline Ceará & 15,1 & 84,9 & 22,9 & 77,1 \\
\hline Rio Grande do Norte & 25,7 & 74,3 & 19,7 & 80,3 \\
\hline Paraíba & 26,9 & 73,1 & 20,1 & 79,9 \\
\hline Pernambuco & 12,5 & 87,5 & 20,7 & 79,3 \\
\hline Alagoas & 12,9 & 87,1 & 17,0 & 83,0 \\
\hline Sergipe & 20,0 & 80,0 & 21,2 & 78,8 \\
\hline Bahia & 17,6 & 82,4 & 22,0 & 78,0 \\
\hline \multicolumn{5}{|l|}{ Sudeste } \\
\hline Minas Gerais & 18,6 & 81,4 & 25,5 & 74,5 \\
\hline Espírito Santo & 17,9 & 82,1 & 24,1 & 75,9 \\
\hline Rio de Janeiro & 16,6 & 83,4 & 18,0 & 82,0 \\
\hline São Paulo & 19,9 & 80,1 & 23,4 & 76,6 \\
\hline \multicolumn{5}{|l|}{ Sul } \\
\hline Paraná & 17,6 & 82,4 & 25,1 & 74,9 \\
\hline Santa Catarina & 15,5 & 84,5 & 28,2 & 71,8 \\
\hline Rio Grande do Sul & 27,5 & 72,5 & 22,8 & 77,2 \\
\hline \multicolumn{5}{|l|}{ Centro Oeste } \\
\hline Mato Grosso do Sul & 18,4 & 81,6 & 27,8 & 72,1 \\
\hline Mato Grosso & 8,4 & 91,6 & 27,8 & 72,2 \\
\hline Goiás & 17,6 & 82,4 & 26,0 & 74,0 \\
\hline Distrito Federal & 30,9 & 69,1 & 27,9 & 72,1 \\
\hline
\end{tabular}

Fonte: Pesquisa Nacional por Amostra de Domicílios 2015(8)

A Figura 1 apresenta a relação entre a prática de atividades físicas e de esporte de acordo com a renda da população estudada. Assim, é possível observar uma relação direta entre a renda per capita mensal por domicílio e a realização de alguma modalidade de atividade. Constata-se que indivíduos com renda maior que 3 salários 
mínimos realizam mais esporte e atividades físicas, sendo os que recebem de 5 a 10 os mais praticantes, e quem menos os realizou foram as pessoas com renda inferior a 3 salários mínimos. Destaca-se que os índices de atividade física são maiores do que os de esporte em todas as variáveis.

Tabela II - Motivação para a prática de esporte e atividade física por renda e sexo. Dados proporcionais de 71.142 brasileiros. Salvador, Bahia, 2015.

\begin{tabular}{|c|c|c|c|c|c|c|c|c|c|c|}
\hline \multirow[t]{2}{*}{ Motivo Principal $\backslash n^{\circ}$ salário (\%) } & \multicolumn{2}{|c|}{ Até 1} & \multicolumn{2}{|c|}{2 a 3} & \multicolumn{2}{|c|}{3 a 5} & \multicolumn{2}{|c|}{5 a 10} & \multicolumn{2}{|c|}{ Acima 10} \\
\hline & A & E & A & E & A & E & A & E & A & E \\
\hline Indicação médica & 17,2 & 9,4 & 9,6 & 6,0 & 11,1 & 8,3 & 11,7 & 6,4 & 9,9 & 8,0 \\
\hline Melhorar ou manter o Desempenho Físico & 22,8 & 17,5 & 28,0 & 21,3 & 27,2 & 25,6 & 26,3 & 31,8 & 27,0 & 22,7 \\
\hline Qualidade de Vida ou Bem-Estar & 39,0 & 23,3 & 41,2 & 26,3 & 44,4 & 35,8 & 50,5 & 39,8 & 49,5 & 44,0 \\
\hline Gosta de Competir & 1,2 & 11,1 & 2,2 & 9,6 & 2,0 & 6,7 & 0,9 & 2,6 & 2,0 & 5,8 \\
\hline Relaxarl Divertir & 15,9 & 34,1 & 15,7 & 31,9 & 12,6 & 20,4 & 8,8 & 17,1 & 11,1 & 17,2 \\
\hline Socializar & 2,5 & 4,0 & 1,7 & 4,8 & 1,6 & 2,3 & 0,4 & 1,4 & 0,3 & 1,3 \\
\hline Outro & 1,4 & 0,5 & 1,5 & 0,1 & 1,0 & 0,8 & 1,4 & 0,9 & 0,3 & 1,1 \\
\hline \multirow[t]{2}{*}{ Motivo Principal por sexo (\%) } & \multicolumn{5}{|c|}{ Masculino } & \multicolumn{5}{|c|}{ Feminino } \\
\hline & \multicolumn{2}{|r|}{ A } & \multicolumn{3}{|c|}{ E } & \multirow{2}{*}{\multicolumn{2}{|c|}{ A }} & & \multicolumn{2}{|l|}{ E } \\
\hline Indicação médica & \multicolumn{2}{|r|}{12,0} & \multicolumn{3}{|c|}{5,6} & 20,5 & & \multicolumn{3}{|c|}{20,2} \\
\hline Melhorar ou manter o Desempenho Físico & \multicolumn{2}{|r|}{27,8} & \multicolumn{3}{|c|}{19,0} & \multicolumn{2}{|r|}{21,1} & \multicolumn{3}{|c|}{22,2} \\
\hline Qualidade de Vida ou Bem-Estar & \multicolumn{2}{|r|}{35,9} & \multicolumn{3}{|c|}{21,7} & \multicolumn{2}{|r|}{44,4} & \multicolumn{3}{|c|}{39,4} \\
\hline Gosta de Competir & \multicolumn{2}{|r|}{3,1} & \multicolumn{3}{|c|}{11,9} & \multicolumn{2}{|r|}{1,1} & \multicolumn{3}{|c|}{3,5} \\
\hline Relaxar/Divertir & \multicolumn{2}{|r|}{17,6} & \multicolumn{3}{|c|}{36,4} & \multirow{2}{*}{\multicolumn{2}{|c|}{$\begin{array}{c}10,5 \\
1,3\end{array}$}} & \multicolumn{3}{|c|}{12,4} \\
\hline Socializar & \multicolumn{2}{|r|}{2,4} & \multicolumn{3}{|c|}{5,0} & & & & 1,6 & \\
\hline Outro & & 1,1 & & 0,4 & & & 1,1 & & 0,7 & \\
\hline
\end{tabular}

A: Atividade Física; E: Esporte; $n^{\circ}$ : Número. Fonte: Pesquisa Nacional por Amostra de Domicilio $2015^{\left({ }^{(}\right)}$

A Tabela III apresenta a relação da prática de atividade por nível de escolaridade. Por meio dela é possível observar que há uma maior proporção de atividades esportivas em todas as variáveis mencionadas. Sobre a prática de esporte nos grupos selecionados, os classificados como Pré-Vestibulares $(56,8 \%)$, Mestrado/Doutorado $(49,4 \%)$, Fundamental $(47 \%)$ e Superior $(35,4 \%)$ aderiram mais a esta prática.

Tratando-se da prática de Atividades Físicas, as pessoas com Mestrado/Doutorado (43,6\%), Superior (29,1\%) e Alfabetização jovens e adultos (22\%) realizam-nas mais que as demais, quando se nota nas duas modalidades que os grupos que mais têm uma vida ativa são os de nível de escolaridade mais elevados. (Tabela III).

Com relação ao estado civil das pessoas que praticaram alguma atividade corporal no Brasil no período da pesquisa, verifica-se na Tabela IV que tanto aquelas que praticaram esporte quanto os que realizaram atividade física são, em sua maioria, solteiras (27,0\%). Ao teste do Qui-Quadrado, a hipótese de que há associação entre estado civil e prática de esporte e atividade física é estatisticamente significativa.

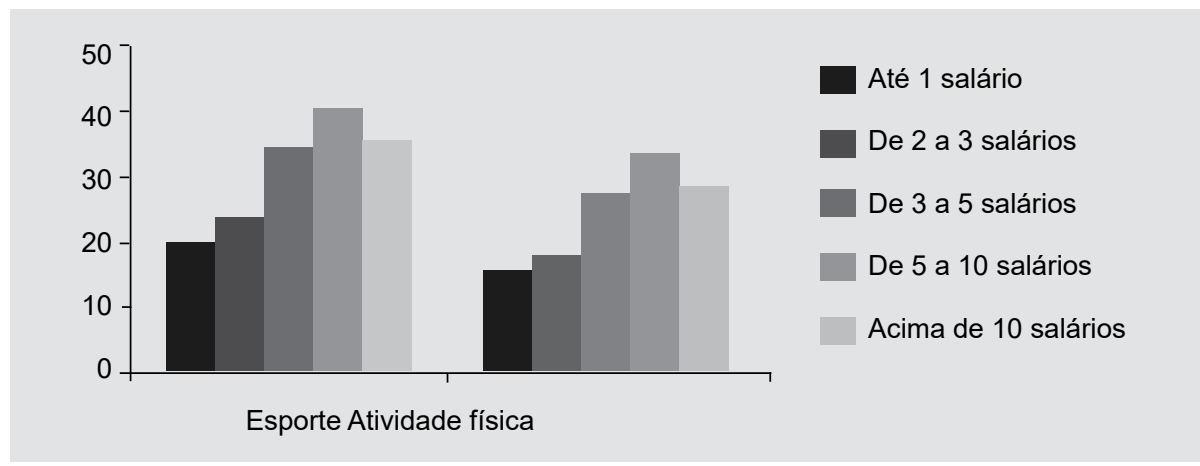

Figura 1 - Prática de esporte e atividade física por renda. Dados proporcionais de 71.142 brasileiros. Salvador, Bahia, 2015.

Fonte: Pesquisa Nacional por Amostra de Domicílios 2015(8) 
Tabela III - Prática de atividade por nível de escolaridade. Dados proporcionais de 71.142 brasileiros. Salvador, Bahia, 2015.

\begin{tabular}{lcc}
\hline \multirow{2}{*}{ Escolaridade } & \multicolumn{2}{c}{ Modalidade da atividade (\%) } \\
\cline { 2 - 3 } Alfabetização jovem e Adulto & Esporte & Atividade Física \\
Fundamental & 23 & 22 \\
Jovens e adultos Fundamental & 47 & 17,8 \\
Jovens e adultos Médio & 25,1 & 14,1 \\
Médio & 31,6 & 18,5 \\
Pré-vestibular & 42,4 & 19 \\
Superior & 56,8 & 17,1 \\
MestradolDoutorado & 35,4 & 29,1 \\
\hline
\end{tabular}

Fonte: Pesquisa Nacional por Amostra de Domicílios 2015(8)

Tabela IV - Prática de esporte e atividade física por estado civil no Brasil. Dados proporcionais de 71.142 brasileiros. Salvador, Bahia, 2017.

\begin{tabular}{lcccccccccc}
\hline Prática/ Estado Civil (\%) & \multicolumn{2}{c}{ Casado } & \multicolumn{2}{c}{ Separado Judicialmente } & \multicolumn{2}{c}{ Divorciado } & \multicolumn{2}{c}{ Viúvo } & \multicolumn{2}{c}{ Solteiro } \\
& E & A & E & A & E & A & E & A & E & A \\
\hline Prática & 18,7 & 15,5 & 17,7 & 17,4 & 21,5 & 22,9 & 12,8 & 16,4 & 27,0 & 18,0 \\
\hline
\end{tabular}

E: Esporte; A: Atividade Física. Fonte: Pesquisa Nacional por Amostra de Domicílios $2015^{(8)}$

\section{DISCUSSÃO}

Os resultados da presente pesquisa revelam que aproximadamente 61,3 milhões de brasileiros realizam atividade física ou esporte, estando essas pessoas, em sua maioria, nas regiões Sul $(40,8 \%)$ e Centro-Oeste $(41,1 \%)$. Dentre os homens, os principais motivos são relaxamento e diversão, enquanto para as mulheres envolvem qualidade de vida e bem-estar, sendo em sua maioria pessoas solteiras que praticam $(27,0 \%)$. O presente estudo também evidenciou maior nível de atividade diretamente proporcional a uma maior escolaridade e renda financeira familiar.

A atividade física é um dos elementos fundamentais para preservação e controle de doenças crônicas como a hipertensão arterial sistêmica (HAS), diabetes mellitus (DM) e obesidade mórbida. A Organização Mundial de Saúde (OMS), no ano de 2010, estimou que baixos níveis de atividade física têm sido apontados como o quarto principal fator de risco das mortes em todo o mundo, assim, o aumento da atividade física diária com a integração de uma rotina de exercícios físicos torna-se um fator determinante para o equilíbrio energético que influenciará diretamente na qualidade de vida e na saúde da população(9).

Ainda não existe um conceito padrão para definir de fato os termos atividade física e exercício, porém entende-se o exercício físico como uma subcategoria da atividade física, que pode ser caracterizado por uma ação planejada, estruturada, repetitiva e proposital no sentido de melhoria ou manutenção de um ou mais componentes de aptidão física. A atividade física, por sua vez, inclui o exercício, envolvendo todo o tipo de movimento corporal produzido pela musculatura esquelética, resultando em gasto energético superior aos níveis de repouso ${ }^{(9)}$. O Ministério da Saúde do Brasil tem levantado estratégias com o intuito de incentivar e desenvolver práticas corporais para melhorar o estilo de vida da população, bem como tratar distúrbios já instalados, como as doenças crônicas não transmissíveis ${ }^{(10)}$.

No entanto, para isso, primeiramente, é necessário conhecer o perfil dos indivíduos que realizam ou não essas práticas corporais, para assim desenvolver métodos de incentivo para a realização dessas práticas em ambas as populações, tanto no intuito de manter como no de promover seu desenvolvimento. Com base nos resultados do atual estudo, constata-se que a maior prevalência de pessoas que praticam atividade física e esporte reside em grandes regiões, com maior percentual no Distrito Federal (30,7\% e 27,9\%). A maior parte da amostra está representada por indivíduos do sexo masculino $(53,9 \%)$, tanto na prática de atividade física quanto de esporte, e a motivação mais frequente para a realização das práticas corporais se deve à busca de relaxamento e qualidade de vida.

Estudos realizados com inquéritos de bases populacionais concluem que praticamente metade da população brasileira não atinge os níveis recomendados (150 minutos semanais) de prática de atividade física, pois apenas 
cerca de aproximadamente $22,5 \%$ a $24,4 \%$ atingiram tais recomendações de atividade física no lazer, sendo tal percentual de prevalência maior entre homens e residentes na área urbana. Referida realidade sugere a necessidade do fortalecimento de ações de promoção de atividade física no país ${ }^{(11,12)}$.

O presente estudo evidencia que o número de pessoas que realizam atividades físicas ou esportes aumenta proporcionalmente com relação ao nível de escolaridade, pois as pessoas com Mestrado/Doutorado apresentaram índices maiores com respectivamente $43,6 \%$ e 49,4\%. Esse fator está relacionado ao acesso à informação, o que auxilia na compreensão da importância das práticas corporais para seu estado de saúde e bem-estar, e estudos relacionam o nível de escolaridade com o sedentarismo e consequentemente com o maior desenvolvimento de doenças crônicas não transmissíveis que estão relacionados com o estilo de vida(13-15).

Outra evidência importante refere-se à prática de esporte e atividade física e a sua associação positiva com a renda mensal, o que pode ser justificado pela maior acessibilidade espacial a locais e instrumentos necessários que propiciam maior engajamento a essas práticas corporais, maior disponibilidade de tempo livre, vivências corporais mais favoráveis à adoção de modos de vida mais ativos, dentre outros ${ }^{(16)}$.

Estudos comprovam que a condição socioeconômica da população tem relação direta não só com seu nível de atividade, como também à maior prevalência de problemas relacionados à saúde, tais como, doenças cardiovasculares e metabólicas (obesidade, diabetes mellitus); renda per capita mensal por domicilio, nível de escolaridade e a atividade laboral. Todos apresentam forte associação com o nível de atividade física e o estado de saúde da população, sendo esses fatores facilitadores ou até de impedimentos na construção de um bom estado de saúde ${ }^{(17,18)}$.

Também se investigou a relação da prática de atividade física e esporte com o estado civil. Alguns estudos têm relacionado a realização de práticas corporais a indivíduos solteiros e justificam que o mesmo acontece pela maior disponibilidade de tempo e da atividade ser realizada também como meio de socialização e lazer ${ }^{(16-18)}$. No presente estudo, os solteiros também realizaram mais atividades corporais $(27,0 \%)$, corroborando a literatura. Outro estudo evidenciou que os jovens do sexo masculino e solteiros praticam mais exercícios físicos ${ }^{(18)}$.

Percebeu-se também no presente estudo, um índice significativo na prática de esporte em pessoas que estudam no ensino fundamental (47\%), que geralmente são um público jovem. A atividade física deve ser estimulada e praticada no meio escolar, visando à promoção de um estilo de vida ativo. No entanto, evidencia-se que apesar de adolescentes possuírem uma elevada percepção sobre hábitos saudáveis de vida e serem ativos, tal fato não tem influenciado de maneira efetiva a prática de atividade física sistematizada, voltada à promoção da saúde e à motivação para adoção desta prática, ocasionando assim o sedentarismo(9).

Estudos afirmam que o ambiente onde o jovem passa a maior parte do tempo pode influenciar positivamente na adoção de posturas que resultam em um estilo de vida saudável e ativo. A escola, então, é considerada um local fundamental para influenciar a adoção de um estilo de vida saudável, desenvolvendo programas efetivos de educação física e estimulando a participação dos alunos, porém é necessário um diagnóstico do perfil da prática de atividades físicas e possíveis riscos da população. Sendo necessária a integração e o trabalho conjunto da escola e das famílias, em termos de comunicação e cooperação, para a adoção de um estilo de vida saudável e a prática de atividades físicas na educação dos filhos, além de modelos positivos e o encorajamento por parte dos pais para o desenvolvimento de estilos de vida mais ativos e saudáveis ${ }^{(9,19)}$.

Acredita-se que a presente pesquisa possa contribuir para as práticas de atividades físicas e esporte no país, visto que resultados apresentados podem servir como base para aprimoramento e implementação de políticas públicas e de estratégias para aumentar a adesão da população às práticas de atividades físicas e esporte ${ }^{(20)}$, essenciais para uma vida mais saudável.

\section{CONCLUSÃO}

As pessoas praticantes de esporte e atividade física no Brasil no ano de 2015, de acordo com a estratificação dos resultados da Pesquisa Nacional de Amostra por Domicílio, caracterizam-se em sua maioria por serem do sexo masculino, com maior nível de escolaridade e renda, solteiros e que buscavam essas atividades para melhorar sua qualidade de vida e bem-estar.

\section{CONFLITOS DE INTERESSE}

Não há conflitos de interesse. 


\section{CONTRIBUIÇÕES}

Sara Souza Pontes, Alana das Mercês Silva e Laíza Muniz de Santana Santos: Elaboração e delineamento do estudo; aquisição, análise e interpretação de dados; Brendo Vitor Nogueira Sousa e Elenilda Farias de Oliveira: Análise e interpretação de dados; redação e/ou revisão do manuscrito.

\section{REFERÊNCIAS}

1. Zamai CA. Impacto das atividades físicas nos indicadores de saúde de sujeitos adultos: programa mexa-se [tese]. São Paulo: Universidade Estadual de Campinas; 2009.

2. World Health Organization. Recomendaciones mundiales sobre actividad física para la salud [Internet]. 2010; [acesso em: 2018 jun 05] Disponível em: http://apps.who.int/iris/bitstream/ handle/10665/44441/9789243599977_spa.pdf;jsessionid=2945859E660C04064D44795158BA4B9D?sequen $\mathrm{ce}=1$

3. Zamai CA, Bankoff ADP. A Ciência e a pesquisa em ação: atividades físicas, saúde e qualidade de vida: pesquisas e relatos de experiências. Jundiaí: Editora Paco; 2016.

4. Carvalho FFB, Nogueira JAD. Práticas corporais e atividades físicas na perspectiva da promoção da saúde na atenção básica. Ciênc Saúde Colet [Internet]. 2016;21(6):1829-38.

5. Malta DC, Moura EC, Castro AM, Cruz DKA, Morais OL Neto, Monteiro CA. Padrão de atividade física em adultos brasileiros: resultados de um inquérito por entrevistas telefônicas, 2006. Epidemiol serv saúde. 2009;18(1):07-16.

6. Knuth AG, Malta DC, Dumith SC, Pereira CA, Morais OI Neto, Temporão JG; et al. Prática de atividade física e sedentarismo em brasileiros: resultados da Pesquisa Nacional por Amostra de Domicílios (PNAD) - 2008. Ciênc Saúde Colet. 2011;16(9):3697-705.

7. Viana KP, Santos $A B$, Luiz RR. Localização das famílias e de saúde auto-avaliada entre adultos brasileiros que vivem nas grandes cidades: Análise multinível. Rev. Saúde Pública e Epidemiol. 2015;7(3):98-107.

8. Instituto Brasileiro de Geografia e Estatística. Prática de esporte e atividade física: 2015. Rio de Janeiro: IBGE; 2017.

9. Pinheiro LE, Trindade RFC, Silva MAI, Machado DRL, Santos CB. Prática de atividade física de escolares do $4^{\circ}$ e $5^{\circ}$ do ensino fundamental da rede pública estadual. Rev Bras Med Esporte. 2017;23(4):308-12.

10. Loch MR, Rodrigues CG, Teixeira DC. Where are the men? Where are the people who live far away? Where are the young?....? Profile of users of physical activity programs offered by Family Health Suport Centers. Rev Bras Ciênc Esporte. 2013;35(4):947-61.

11. Silva ICM, Knuth AG, Mielke GI, Azevedo MR, Gonçalves H, Hallal PC. Trends in leisure-time physical activity in a Southern Brazilian city: 2003-2010. J Phys Act Health. 2014;11(7):1313-17.

12. Mielke GI, Hallal PC, Rodrigues GBA, Szwarcwald CL, Santos FV, Malta DC. Prática de atividade física e hábito de assistir à televisão entre adultos no Brasil: Pesquisa Nacional de Saúde 2013. Epidemiol Serv Saúde. 2015;24(2):277-86.

13. Freire RS, Lélis FLO, Fonseca JA Filho, Nepomuceno MO, Silveira MF. Prática regular de atividade física: estudo de base populacional no norte de Minas Gerais, Brasil. Rev Bras Med Esporte. 2014;20(5):345-49.

14. Tassitano RM, Mielke GI, Carvalho WRG, Oliveira MM, Malta DC. Tendência temporal de indicadores da prática de atividade física e comportamento sedentário nas capitais da Região Nordeste do Brasil: 20062013. Rev Bras Ativ Fís Saúde. 2015;20(2):152-64.

15. Malta DC, Andrade SSA, Santos MAS, Rodrigues GBA, Mielke GI. Tendências dos indicadores de atividade física em adultos: conjunto de capitais do Brasil 2006-2013. Rev Bras Ativ Fís Saúde. 2015;20(2):141-51.

16. Karusisi N, Thomas F, Méline J, Chaix B. Spatial accessibility to specific sport facilities and corresponding sport practice: the RECORD Study. Int J Behav Nutr Phys Act. 2013;10(48):1-10.

17. Bauman AE, Reis RS, Sallis JF, Wells JC, Loos RJF, Martin BW. Correlates of physical activity: why are some people physically active and others not? Lancet. 2012;380(9838):258-71. 
18. Mielke GI, Hallal PC, Malta DC, Lee IM. Time trends of physical activity and television viewing time in Brazil: 2006-2012. Int J Behav Nutr Phys Act. 2014;11:101.

19. Gallegos AG, Extremera AB, Sánchez-Fuentes JA, Martinez-Molina M. Motivational profiles of autonomy support, self-determination, satisfaction, importance of physical education and intention to partake in leisure time physical activity. Cuad Psicol Deporte. 2014;14(2):59-70.

20. Gonçalves MP, Alchieri JC. Motivação à prática de atividades físicas: um estudo com praticantes não-atletas. Psico-USf. 2010;15(1):125-34.

Endereço para correspondência:

Sarah Souza Pontes

Universidade Federal da Bahia - UFBA

Rua Augusto Viana, s/n - Palácio da Reitoria

Bairro: Canela

CEP: 40110-909 - Salvador - BA - Brasil

E-mail: sarahpontes@gmail.com

Como citar: Pontes SS, Silva AM, Santos LMS, Sousa BVN, Oliveira EF. Práticas de atividade física e esporte no Brasil. Rev Bras Promoç Saúde. 2019;32:8406. 